\title{
Slow Collateral Flow Is Associated with Thrombus Extension in Patients with Acute Large-Artery Occlusion
}

\author{
(D) R. Zhang, (D) Y. Zhou, (DS. Yan, (DS. Zhang, (D) X. Ding, and (D). Lou
}

\begin{abstract}
BACKGROUND AND PURPOSE: It is still poorly understood about the dynamic changes of the thrombus after intravenous thrombolysis and how the remaining thrombus affects clinical outcome in human stroke. Collateral flow was assumed to help to deliver endo/ exogenous tissue-type plasminogen activator to the clot. We aimed to analyze the impact of collateral flow on the dynamic changes of the thrombus in patients with acute large-artery occlusion who received intravenous thrombolysis.
\end{abstract}

MATERIALS AND METHODS: We reviewed consecutive patients with acute ischemic stroke with M1 segment or distal internal carotid artery occlusion who underwent multimodal MR imaging or CT perfusion before and 24 hours after intravenous thrombolysis without recanalization. Patients were divided into 3 groups (thrombus extension, shortening, and no change) according to thrombus-length change between baseline and 24 hours. Collateral flow was measured with arrival time delay and the collateral scoring system. Poor outcome was defined as a 3-month modified Rankin Scale score of $\geq 3$.

RESULTS: Among 51 patients, 18 (35.3\%) had thrombus extension, 14 (27\%) had thrombus shortening, and 19 (37.3\%) had thrombus without change. Arrival time delay was independently associated with thrombus extension $(\mathrm{OR}=1.499 ; 95 \% \mathrm{Cl}, 1.053-2.135 ; P=.025)$. Similarly, the collateral score on the peak artery phase was independently associated with thrombus extension ( $\mathrm{OR}=0.456 ; 95 \% \mathrm{Cl}, 0.211-0.984 ; P=.045)$, whereas baseline National Institutes of Health Stroke Scale score $(\mathrm{OR}=0.768 ; 95 \% \mathrm{Cl}, 0.614-0.961 ; P=.021)$ and baseline thrombus length (OR $=$ $1.193 ; 95 \% \mathrm{Cl}, 1.021-1.394 ; P=.026)$ were associated with thrombus shortening. All patients with thrombus extension had poor outcomes.

CONCLUSIONS: Slow collateral flow was related to thrombus extension in patients with large-artery occlusion without recanalization after intravenous thrombolysis.

ABBREVIATIONS: ATD = arrival time delay; $\mathrm{dGE}-\mathrm{TI}=$ delayed gadolinium-enhanced TT; IVT $=$ intravenous thrombolysis; $\mathrm{LAO}=$ large-artery occlusion; $\mathrm{tMIP}=$ temporally fused MIP

$\mathbf{P}$ revious studies have demonstrated that characteristics of thrombus, especially thrombus length, were closely related to the rate of recanalization, clinical outcome after intravenous thrombolysis (IVT), ${ }^{1}$ and even stroke etiology in patients with acute ischemic stroke with large-artery occlusion (LAO). ${ }^{2,3}$ However, the dynamic changes of the thrombus after IVT and how the remaining thrombus affects clinical outcome in human stroke are still poorly understood.

Received November 27, 2017; accepted after revision February 6, 2018.

From the Departments of Neurology (R.Z., Y.Z., S.Y., S.Z., M.L.) and Radiology (X.D.), Second Affiliated Hospital of Zhejiang University, School of Medicine, Hangzhou, China; and Zhejiang University Brain Research Institute (M.L.), Hangzhou, China.

This work was support by the National Natural Science Foundation of China (81471170 and 81622017), the National Key Research and Development Program of China (2016YFC1301500), and the National Natural Science Foundation of China (81400946).

Please address correspondence to Min Lou, MD, PhD, Department of Neurology, Second Affiliated Hospital of Zhejiang University, School of Medicine, 88 Jiefang Rd, Hangzhou, 310009, China; e-mail: loumingxc@vip.sina.com, Im99@zju.edu.cn

- Indicates open access to non-subscribers at www.ajnr.org

http://dx.doi.org/10.3174/ajnr.A5614
Collateral flow was assumed to help deliver endo-/exogenous tissue-type plasminogen activator to the clot. Indeed, evidence has shown that good collaterals were often related to short baseline thrombus. ${ }^{4}$ Our previous study also demonstrated that rapid collaterals accomplished high recanalization rates after IVT. ${ }^{5} \mathrm{We}$ thus hypothesized that collateral velocity might have an impact on dynamic changes of the thrombus.

With rapid development of in vivo thrombus imaging techniques, baseline characteristics of thrombus have been widely studied. Delayed gadolinium-enhanced T1 (dGE-T1), which can delineate the distal end of the thrombus and avoid blooming artifacts of the susceptibility vessel sign, was proved a useful tool for accurate measurement of thrombus length when combined with time-of-flight MR angiography. ${ }^{1}$ Similarly, 4D CT angiography, derived from CT perfusion, can not only outline intracranial thrombi better than conventional single-phase $\mathrm{CTA}^{6}$ but also evaluate collateral flow with increased sensitivity. ${ }^{\text {? }}$

Therefore, we designed this study with the following objec- 
tives: 1) to assess the change of thrombus length in patients with LAO without recanalization at 24 hours after IVT detected on dGE-T1 and 4D-CTA derived from CTP, 2) to evaluate the relationship between the changing of thrombus length and collaterals, and 3) to find the influencing factors of different changing patterns of thrombus length.

\section{MATERIALS AND METHODS \\ Ethics Statement}

The protocol of MR imaging/CT-guided intravenous thrombolysis was approved by our local human ethics committee. All clinical investigations were conducted according to the principles expressed in the Declaration of Helsinki. Informed consent was obtained for all patients.

\section{Patient Selection}

We retrospectively reviewed our prospectively collected data base for consecutive patients with acute ischemic stroke who received IVT alone between June 2009 and March 2017. We enrolled patients using the following criteria: 1) They had a diagnosis of acute ischemic stroke confirmed by diffusion-weighted imaging or CTP, 2) received IVT within 6 hours from symptom onset, 3) had occlusion of middle cerebral artery M1 segment or distal internal carotid artery on baseline TOF-MRA or CTA, and 4) underwent follow-up CTP or multimodal MR imaging at 24 hours with no confirmed recanalization, which was defined as 0 or 1 of the Arterial Occlusive Lesion scale (details in the "Evaluation of Outcome" section). Patients with poor image quality because of motion artifacts were excluded.

\section{Imaging Protocols}

Subjects underwent MR imaging on a 3T system (Signa Excite HD; GE Healthcare, Milwaukee, Wisconsin) equipped with an 8 -channel phased array head coil. The DWI sequence was used to measure the infarct volume $(\mathrm{TR}=4000 \mathrm{~ms}$, $\mathrm{TE}=69.3 \mathrm{~ms}$, b-value $=1000 \mathrm{~s} / \mathrm{mm}^{2}$, slice thickness $=5.0 \mathrm{~mm}$, and interslice gap $=1.0 \mathrm{~mm}$ ). TOF-MRA consisted of 3 slabs with $\mathrm{TR}=20 \mathrm{~ms}$, $\mathrm{TE}=3.2 \mathrm{~ms}$, flip angle $=15^{\circ}$, and slice thickness $=1.4 \mathrm{~mm}$. Fluid-attenuated inversion recovery parameters were $\mathrm{TR}=9000$ $\mathrm{ms}, \mathrm{TE}=150 \mathrm{~ms}, \mathrm{TI}=2250 \mathrm{~ms}$, and slice thickness $=5.0 \mathrm{~mm}$. PWI was performed using the standard bolus passage of contrast method by injecting gadolinium $(0.1 \mathrm{mmol} / \mathrm{kg}$ dose via a power injector). PWI parameters were the following: TR $=1500 \mathrm{~ms}$, $\mathrm{TE}=30 \mathrm{~ms}$, and slice thickness $=5.0 \mathrm{~mm}$. Conventional $\mathrm{T} 1$ parameters were $\mathrm{TR}=1900 \mathrm{~ms}, \mathrm{TE}=25 \mathrm{~ms}$, and slice thickness $=5.0 \mathrm{~mm}$.

CTP was performed on a dual-source 64-slice CT scanner (Somatom Definition Flash; Siemens, Erlangen, Germany), including a nonenhanced head CT scan $(120 \mathrm{kV}, 320 \mathrm{~mA}$, contiguous $5-\mathrm{mm}$ axial slices), and volume perfusion CT (100 $\mathrm{mm}$ in the z-axis, 4-second delay after start of contrast medium injection, 74.5-second total imaging duration, $80 \mathrm{kV}, 120 \mathrm{~mA}$, effective dose $=3.68 \mathrm{mSv}$, slice thickness $=10 \mathrm{~mm}$, collimation $=32 \times$ $1.2 \mathrm{~mm}$ ). Volume perfusion CT consisted of 26 consecutive spiral acquisitions of the brain. A 60-mL bolus of contrast medium (iopamidol, Imeron; Bracco, Milan, Italy) was used at a flow rate of 6 $\mathrm{mL} / \mathrm{s}$, followed by a $20-\mathrm{mL}$ saline chaser at $6 \mathrm{~mL} / \mathrm{s}$.

\section{Imaging Analysis}

Two neurologists (R.Z. and Y.Z.), blinded to patients' clinical information, independently assessed the thrombus length on dGE-T1 and CTA, respectively, as described in our previous study. ${ }^{1}$ For multimodal MR imaging, we coregistered the source images of TOF-MRA and dGE-T1 with MRIcron NIfTI viewer (http://www.mccauslandcenter.sc.edu/crnl/) because the distal vessels beyond the clot were clearly visible on dGE-T1. We then manually measured the distance between the proximal and distal ends of the occlusion, orienting along the central axis of the artery, as thrombus length. Similarly, we reconstructed 4D-CTA from CTP with commercially available software (MIStar; Apollo Medical Imaging Technology, Melbourne, Victoria, Australia); and temporally fused MIP (tMIP) datasets were created for the identification of the proximal and distal ends of clots. Thrombus length was measured along the central axis of the artery. For patients who underwent different imaging at baseline and 24 hours, we coregisterred the CTA images to the dGE-T1 images on MIStar software and calculated the thrombus length on the coregisterred CTA images. Thrombus extension was defined as (24-Hour Thrombus Length - Baseline Thrombus Length) / Baseline Thrombus Length $>30 \%$. Thrombus shortening was defined as (Baseline Thrombus Length - 24-Hour Thrombus Length) / Baseline Thrombus Length $>30 \%$. The others $[-30 \% \leq$ (Baseline Thrombus Length -24 -Hour Thrombus Length) $\leq 30 \%$ ] were classified as thrombus without change.

Measurement of collateral flow velocity was quantified on PWI- or CTP-derived subtraction images, also described in our previous study. ${ }^{5}$ Briefly, the artery that crossed the Sylvian fissure and was located at the end of the MCA M1 segment of each hemisphere was used to generate the arterial input function curve for the ischemic and contralateral hemispheres, respectively. The first time point when contrast reached the selected artery was then identified on the arterial input function curve for the ischemic and contralateral hemispheres, respectively. The arrival time delay (ATD) was defined as the time difference between these 2 time points.

The status of the leptomeningeal collateral circulation was evaluated by the scoring system of Tan et al. ${ }^{8}$ In short, the collateral score was determined according to the following rules: 0 , absent collaterals; 1 , collateral filling $\leq 50 \%$ of the occluded territory; 2 , collateral filling $\geq 50 \%$ but $\leq 100 \%$ of the occluded territory; and 3, collateral filling $100 \%$ of the occluded territory. Collaterals were evaluated on PWI- or CTP-derived subtraction images. Peak artery phase and tMIP reconstructions from PWI or CTP images that fuse contrast opacification across the duration were obtained. The collateral scores on peak artery phase images were recorded as peak scores of collaterals, which reflect both the velocity and extent of collaterals. Moreover, the collateral scores on tMIP were recorded as the tMIP scores of collaterals, which reflect the extent of the collaterals.

\section{Evaluation of Outcome}

We used the Arterial Occlusive Lesion scale (grade 0, complete occlusion of the target artery; grade 1, incomplete occlusion or partial local recanalization at the target artery with no distal flow; grade 2, incomplete occlusion or partial local recanalization at the 
Table 1: Characteristics of patients with different changing patterns of thrombus length

\begin{tabular}{|c|c|c|c|c|}
\hline & $\begin{array}{l}\text { Thrombus Shortening } \\
\qquad(n=14)\end{array}$ & $\begin{array}{c}\text { Thrombus without } \\
\text { Change }(n=19)\end{array}$ & $\begin{array}{l}\text { Thrombus Extension } \\
\qquad(n=18)\end{array}$ & $P$ Value \\
\hline Age (yr) (mean) & $62.79 \pm 14.74$ & $67.53 \pm 13.47$ & $70.44 \pm 11.63$ & .275 \\
\hline Women (No.) (\%) & $4(28.6)$ & $7(36.8)$ & $9(50)$ & .480 \\
\hline \multicolumn{5}{|l|}{ Comorbid conditions } \\
\hline Atrial fibrillation (No.) (\%) & $7(50)$ & $9(47.4)$ & $10(55.6)$ & .938 \\
\hline Hypertension (No.) (\%) & $10(71.4)$ & $15(78.9)$ & $16(88.9)$ & .502 \\
\hline Diabetes (No.) (\%) & $1(7.1)^{\prime}$ & $2(10.5)$ & $5(27.8)$ & .245 \\
\hline Hyperlipidemia (No.) (\%) & $10(71.4)$ & $10(52.6)$ & $8(44.4)$ & .316 \\
\hline Smoking (No.) (\%) & $7(50)$ & $10(52.6)$ & $6(33.3)$ & .461 \\
\hline \multicolumn{5}{|l|}{ Clinical variables } \\
\hline NIHSS score (range) & $6.5(4.5-11.75)$ & $11(7-16)$ & $16(13-18)$ & $<.001$ \\
\hline Onset to imaging (mean) (min) & $201.43 \pm 98.09$ & $233.58 \pm 139.42$ & $175.28 \pm 67.20$ & .263 \\
\hline Previous antiplatelet use (No.) (\%) & $2(14.3)$ & $4(21.1)$ & $1(6.6)$ & .406 \\
\hline Platelets $\left(10^{9} / \mathrm{L}\right)$ (mean) & $189.50 \pm 84.43$ & $200.89 \pm 98.60$ & $179.94 \pm 42.89$ & .723 \\
\hline INR (mean) & $1.07 \pm 0.14$ & $1.02 \pm 0.07$ & $1.01 \pm 0.08$ & .229 \\
\hline Baseline thrombus length (range) (mm) & $12.99(6.93-18.74)$ & $8.0(3.3-11.10)$ & $13.31(6.35-17.59)$ & .116 \\
\hline Baseline ATD (range) (sec) & $3.24(1.71-5.42)$ & $2.77(0.90-4.48)$ & $7.24(3.52-9.18)$ & .001 \\
\hline Peak score of collaterals (range) & $3(1-3)$ & $3(1-3)$ & $1(0-2)$ & .001 \\
\hline tMIP score of collaterals (range) & $3(1-3)$ & $3(2-3)$ & $2(1-3)$ & .176 \\
\hline Poor outcome (No.) (\%) & $7(50)$ & $16(84.2)$ & $18(100)$ & .001 \\
\hline
\end{tabular}

Note:-INR indicates international normalized ratio.

Table 2: Logistic regression for prediction of thrombus extension and shortening, including ATD in the model

\begin{tabular}{|c|c|c|c|}
\hline & OR & $95 \% \mathrm{Cl}$ & $\begin{array}{c}P \\
\text { Value }\end{array}$ \\
\hline \multicolumn{4}{|l|}{$\begin{array}{l}\text { For thrombus extension (set without } \\
\text { change as reference) }\end{array}$} \\
\hline NIHSS score & 1.195 & $0.978-1.460$ & .081 \\
\hline Baseline thrombus length (mm) & 0.981 & $0.853-1.129$ & .790 \\
\hline Baseline ATD (sec) & 1.499 & $1.053-2.135$ & .025 \\
\hline \multicolumn{4}{|l|}{$\begin{array}{l}\text { For thrombus shortening (set without } \\
\text { change as reference) }\end{array}$} \\
\hline NIHSS score & 0.768 & $0.614-0.961$ & .021 \\
\hline Baseline thrombus length (mm) & 1.193 & $1.021-1.394$ & .026 \\
\hline Baseline ATD (sec) & 0.955 & $0.647-1.410$ & .818 \\
\hline
\end{tabular}

Table 3: Logistic regression for prediction of thrombus extension and shortening, including baseline peak score of collaterals in the model

\begin{tabular}{|c|c|c|c|}
\hline & OR & $95 \% \mathrm{Cl}$ & $\begin{array}{c}P \\
\text { Value }\end{array}$ \\
\hline \multicolumn{4}{|l|}{$\begin{array}{l}\text { For thrombus extension (set without } \\
\text { change as reference) }\end{array}$} \\
\hline NIHSS score & 1.146 & $0.935-1.404$ & .189 \\
\hline Baseline thrombus length (mm) & 1.042 & $0.915-1.186$ & .537 \\
\hline Baseline peak score of collaterals & 0.456 & $0.211-0.984$ & .045 \\
\hline \multicolumn{4}{|l|}{$\begin{array}{l}\text { For thrombus shortening (set without } \\
\text { change as reference) }\end{array}$} \\
\hline NIHSS score & 0.742 & $0.580-0.950$ & .018 \\
\hline Baseline thrombus length (mm) & 1.220 & $1.037-1.435$ & .016 \\
\hline Baseline peak score of collaterals & 0.899 & $0.316-2.557$ & .841 \\
\hline
\end{tabular}

target artery with any distal flow; and grade 3, complete recanalization and restoration of the target artery with any distal flow) to define recanalization or no recanalization based on the presence (grade 2 or 3 ) or absence (grade 0 or 1 ) of any downstream flow on 24-hour TOF-MRA or 4D-CTA. Clinical outcome at 3 months was assessed with the mRS and dichotomized into good outcome $(0-2)$ and poor outcome (3-6).

\section{Statistical Analysis}

The patients were trichotomized according to the changing pattern of the thrombus length. A 1-way analysis of variance or
Kruskal-Wallis test was used among multiple groups. Variables with a $P<.05$ in univariate regression analyses and baseline thrombus length were included in the multinomial logistic regression. We performed all analyses blinded to the participant identifying information. Statistical significance was set at a probability value of $<.05$. All statistical analyses were performed with an SPSS package (Version 14.0 for Windows; IBM, Armonk, New York).

\section{RESULTS}

Fifty-one patients were included in the final analysis. The median age was 67 years (mean, $67 \pm 13$ years; range, $40-94$ years) and 20 (39.2\%) were women. The median baseline NIHSS score was 13 (interquartile range, 7-17). The mean time from onset to imaging was $204 \pm 108$ minutes. The median thrombus length was 11.10 $\mathrm{mm}$ (interquartile range, $6.05-15.90 \mathrm{~mm}$ ) at baseline and 8.74 $\mathrm{mm}$ (interquartile range, $3.65-20.30 \mathrm{~mm}$ ) at 24 hours. The median ATD was 3.63 seconds (interquartile range, 1.90-7.05 seconds) at baseline and 3.52 seconds (interquartile range, $1.29-6.30$ seconds) at 24 hours. Thirty-one $(60.8 \%)$ patients underwent CTP, while 20 (39.2\%) patients underwent MR imaging.

Follow-up scans at 24 hours revealed thrombus extension in $18(35.3 \%)$ patients, thrombus shortening in $14(27.5 \%)$ patients, and thrombus with no change in $19(37.3 \%)$ patients. As Table 1 shows, patients with thrombus extension had longer ATDs (7.24 versus 3.24 versus 2.77 seconds, $P=.001, P<.001)$ and a lower peak score of collaterals ( 1 versus 3 versus $3, P=.002, P=.004$ ) compared with those with thrombus shortening and no change. The tMIP scores of collaterals were comparable among the 3 groups ( 2 versus 3 versus $3, P=.283, P=.150$ ). Patients with thrombus shortening had lower NIHSS scores compared with those with thrombus extension and no change ( 6.5 versus 16 versus $11, P<.001, P=.022)$. Moreover, the rate of poor outcome was significantly higher in patients with thrombus extension than in those with thrombus shortening (100\% versus $50 \%, P=.001)$.

As Table 2 shows, a multinomial logistic regression model, including baseline NIHSS score, baseline thrombus length, and ATD, revealed that ATD was independently associated with 
thrombus extension. Also, the peak score of collaterals but not the tMIP score of collaterals was independently associated with thrombus extension after adjusting for baseline NIHSS score and baseline thrombus length (Tables 3 and 4). Baseline NIHSS score and baseline thrombus length were the independent factors associated with thrombus shortening. The Figure is an example showing correlation between thrombus extension and slow collaterals.

Receiver operating characteristic analysis revealed an acceptable value of ATD for predicting thrombus extension, as a refer-

Table 4: Logistic regression for prediction of thrombus extension and shortening, including baseline tMIP score of collaterals in the model

\begin{tabular}{|c|c|c|c|}
\hline & OR & $95 \% \mathrm{Cl}$ & $\begin{array}{c}P \\
\text { Value }\end{array}$ \\
\hline \multicolumn{4}{|l|}{$\begin{array}{l}\text { For thrombus extension (set without } \\
\text { change as reference) }\end{array}$} \\
\hline NIHSS score & 1.162 & $0.965-1.399$ & .113 \\
\hline Baseline thrombus length (mm) & 1.076 & $0.953-1.216$ & .238 \\
\hline Baseline tMIP score of collaterals & 0.759 & $0.341-1.690$ & .499 \\
\hline \multicolumn{4}{|l|}{$\begin{array}{l}\text { For thrombus shortening (set without } \\
\text { change as reference) }\end{array}$} \\
\hline NIHSS score & 0.719 & $0.563-0.917$ & .008 \\
\hline Baseline thrombus length (mm) & 1.210 & $1.039-1.409$ & .014 \\
\hline Baseline tMIP score of collaterals & 0.571 & $0.212-1.536$ & .267 \\
\hline
\end{tabular}

ence of thrombus without change (area under the curve, 0.804; $95 \%$ confidence interval, $0.663-0.945 ; P=.002$ ). The cutoff point of ATD was 4.61 seconds, and this yielded a sensitivity of $77.2 \%$ and a specificity of $78.9 \%$.

Receiver operating characteristic analysis also showed that baseline thrombus length can predict thrombus shortening, as a reference of thrombus without change (area under the curve, $0.733 ; 95 \%$ confidence interval, $0.551-0.916 ; P=.024)$. The cutoff point of baseline thrombus length was $12.5 \mathrm{~mm}$, and this yielded a sensitivity of $71.4 \%$ and a specificity of $84.2 \%$.

We also performed subanalysis for patients who underwent different imaging techniques for evaluation of collateral flow. In patients who underwent CTP, those with thrombus extension tended to have longer ATDs ( 7.24 versus 3.68 versus 4.48 seconds, $P=.006$, $P=.109)$ and a lower peak score of collaterals (1 versus 3 versus 2, $P=.002, P=.124)$ compared with those with thrombus shortening and no change; these findings were in accordance with our main results. In patients who underwent MR perfusion, those with thrombus extension tended to have longer ATDs (5.87 versus 3.06 versus 1.39 seconds, $P=.352, P=.076)$ and a lower peak score of collaterals (1 versus 2 versus $3, P=.352, P=.054$ ) compared with those with thrombus shortening and no change. The MR perfusion subgroup analysis was not significant due to the small sample size.

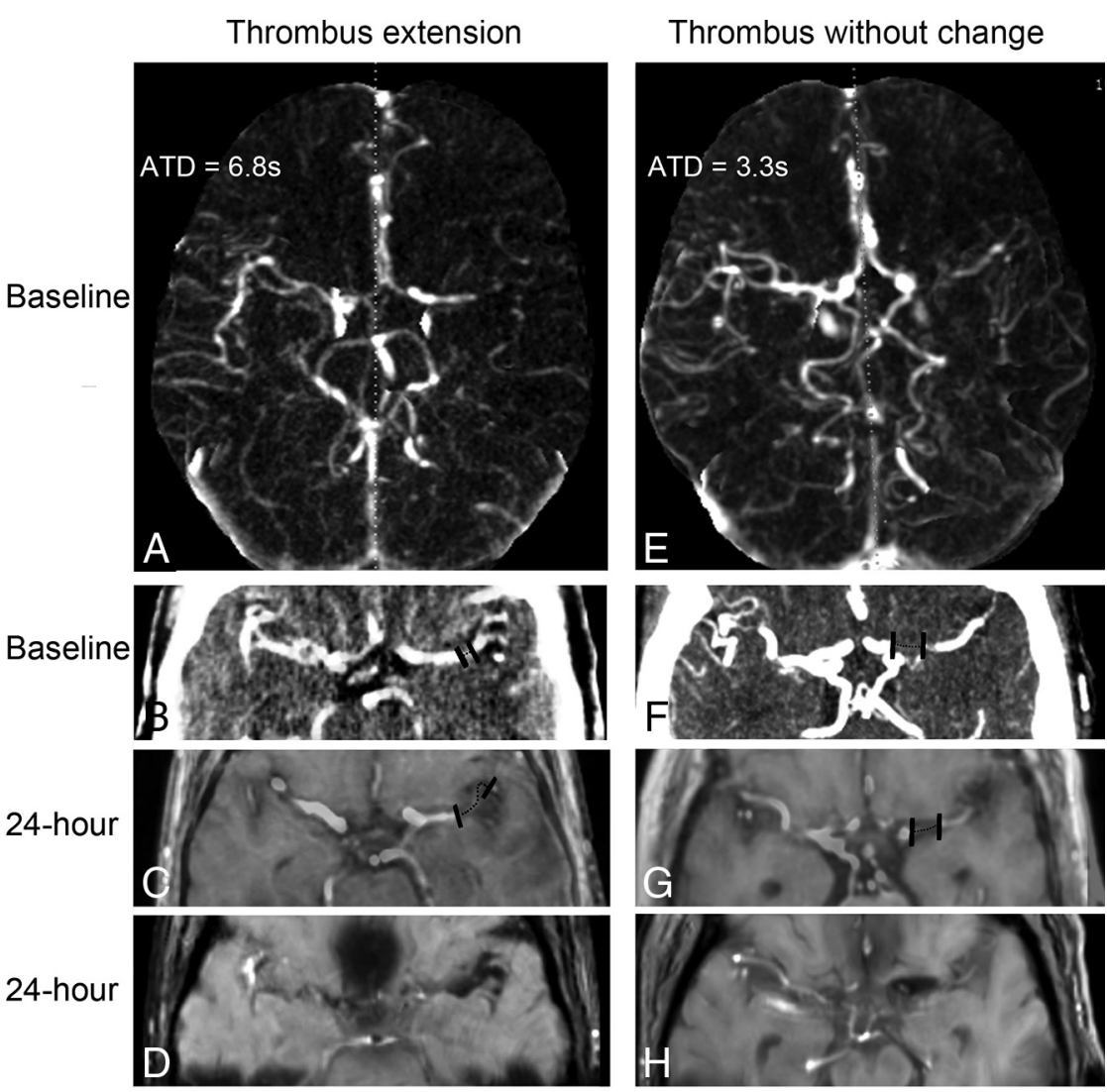

FIGURE. Representative examples of patients with different thrombus-changing patterns. $A-D$, Examples of thrombus extension. A, Arterial peak phase of 4D-CTA shows the collateral flow at baseline, with an arrival time delay of 6.8 seconds. Thrombus length was measured on TMIP of 4D-CTA $(B)$ and a merged image of dGE-Tl and TOF-MRA (C), which was $4.0 \mathrm{~mm}$ at baseline and $18.8 \mathrm{~mm}$ at 24 hours. $D$, Magnitude image at 24 hours also shows the thrombus. $E-H$, Examples of thrombus without change. $E$, The arterial peak phase of 4D-CTA shows the collateral flow at baseline with an ATD of 3.3 seconds. Thrombus length was measured on TMIP of 4D-CTA $(F)$ and the merged image of dGE-Tl and TOF-MRA (G), which was $11.2 \mathrm{~mm}$ at baseline and $10.9 \mathrm{~mm}$ at 24 hours. $H$, Magnitude image at 24 hours also shows the thrombus.

\section{DISCUSSION}

In this study, we illustrated the change pattern of thrombus in patients with LAO without recanalization at 24 hours after IVT and revealed that the rate of thrombus extension and shortening was $35.3 \%$ and $27 \%$, respectively. Slow collateral filling was associated with thrombus extension, whereas a low NIHSS score and long thrombus length were related to thrombus shortening. Moreover, all patients with thrombus extension had poor outcome at 3 months.

Rapid collaterals had high rates of recanalization after IVT and endovascular therapy. ${ }^{5,7}$ Our study further confirmed that slow collateral filling could be related to thrombus extension if recanalization was not achieved. Slow collateral flow represents the stasis of blood flow at the occlusion site, which may cause platelet aggregation, local accumulation of inflammatory mediators, leukocyte activation, and endothelial dysfunction. ${ }^{9}$ Moreover, slower collateral flow can result in low shear stress to the thrombus because collateral flow velocity is a surrogate for the pressure drop across the collateral vessels and the occlusion site. ${ }^{5}$ These hemodynamic changes thus create an environment conducive to the formation of a new clot and are therefore associated with the ex- 
tension of the thrombus. Most interesting, collateral extent was not associated with thrombus extension. Extensive collaterals do not necessarily re-establish arterial blood flow but may serve as pathways for venous flow diversion known as cerebral venous steal, due to the increased inflow resistance and focal compression after infarction. ${ }^{10}$ Therefore, the collateral flow may be diverted to veins; this diversion results in a decreased effect on the distal end of the clot. Overall, collateral status could interact with thrombus extension and reconstruct the local angioarchitecture.

It is interesting that we found that thrombus shortening was related to baseline long thrombus and a low NIHSS score. The mild symptoms in patients with relatively long thrombi may suggest good compensated perfusion of the affected brain tissue. Recent preclinical research has shown that better perfusion provided more tPA at the distal end of thrombus, which was helpful to the disruption of the thrombus during the initiation of IVT to 24 hours posttreatment. ${ }^{11}$

Approximately $6 \%-25 \%$ of patients with LAO still fail to achieve successful recanalization and have disabilities, though advances in the device technology of endovascular therapy have improved the speed and efficacy of recanalization in patients with LAO. ${ }^{12,13}$ A previous study has demonstrated that unexplained early neurologic deterioration occurring after thrombolysis was independently associated with the susceptibility vessel sign extension in patients without recanalization. ${ }^{14}$ In our study, no patients with thrombus extension had good outcomes at 3 months. Our findings indicate that collateral failure might act not only as a cause but possibly an inevitable secondary consequence of thrombus extension and expansion of infarction. Therefore, prevention of thrombus extension by early administration of antithrombotics and early improvement of collateral circulation is of equal importance for patients with $\mathrm{LAO}$ without recanalization.

Our study had several limitations. First, it had a retrospective design in a single stroke center (the Second Affiliated Hospital of Zhejiang University, School of Medicine) and a relatively small number of patients. This feature might have a potential risk of selection bias, though data were prospectively established using a stroke registry and standard CTP/MR imaging protocol. Second, thrombus length measured by dGE-T1 and 4D-CTA may be potentially different, though we used coregistration to minimize the discrepancy. Third, we included only patients with M1 segment or distal ICA occlusion to reduce the heterogeneity. It would be clinically important to investigate the thrombus-changing patterns at other sites regarding the various hemodynamics in different vessels. Fourth, our observation that thrombus extension is associated with slow collateral flow could not prove causality. Further studies with time-series data would be needed to demonstrate the causal relationship.

\section{CONCLUSIONS}

With in vivo thrombus imaging techniques, we first proved that slow collateral flow was related to thrombus extension in patients with LAO without recanalization after IVT. Meanwhile, no patients with thrombus extension had good outcomes at 3 months, which highlights the importance of prevention of thrombus extension and early improvement of collateral circulation in patients without recanalization.

Disclosures: Min Lou-RELATED: Grant: National Natural Science Foundation of China, National Key Research and Development Program of China, Comments: This work was support by the National Natural Science Foundation of China (81471170 and 81622017), the National Key Research and Development Program of China (2016YFC1301500), and the National Natural Science Foundation of China (81400946).* *Money paid to the institution.

\section{REFERENCES}

1. Yan S, Chen Q, Xu M, et al. Thrombus length estimation on delayed gadolinium-enhanced T1. Stroke 2016;47:756-61 CrossRef Medline

2. Kang DW, Jeong HG, Kim DY, et al. Prediction of stroke subtype and recanalization using susceptibility vessel sign on susceptibility-weighted magnetic resonance imaging. Stroke 2017;48: 1554-59 CrossRef Medline

3. Zhang R, Zhou Y, Liu C, et al. Overestimation of susceptibility vessel sign: a predictive marker of stroke cause. Stroke 2017;48:1993-96 CrossRef Medline

4. Qazi EM, Sohn SI, Mishra S, et al. Thrombus characteristics are related to collaterals and angioarchitecture in acute stroke. Can J Neurol Sci 2015;42:381-88 CrossRef Medline

5. Zhang S, Zhang X, Yan S, et al. The velocity of collateral filling predicts recanalization in acute ischemic stroke after intravenous thrombolysis. Sci Rep 2016;6:27880 CrossRef Medline

6. Frölich AM, Schrader D, Klotz E, et al. 4D CT angiography more closely defines intracranial thrombus burden than single-phase CT angiography. AJNR Am J Neuroradiol 2013;34:1908-13 CrossRef Medline

7. Zhang S, Chen W, Tang $\mathrm{H}$, et al. The prognostic value of a fourdimensional CT angiography-based collateral grading scale for reperfusion therapy in acute ischemic stroke patients. PLoS One 2016;11:e0160502 CrossRef Medline

8. Tan JC, Dillon WP, Liu S, et al. Systematic comparison of perfusion-CT and CT-angiography in acute stroke patients. Ann Neurol 2007;61:533-43 CrossRef Medline

9. Diamond SL. Systems analysis of thrombus formation. Circ Res 2016;118:1348-62 CrossRef Medline

10. Pranevicius O, Pranevicius M, Pranevicius H, et al. Transition to collateral flow after arterial occlusion predisposes to cerebral venous steal. Stroke 2012;43:575-79 CrossRef Medline

11. Kim DE, Kim JY, Schellingerhout D, et al. Quantitative imaging of cerebral thromboemboli in vivo: the effects of tissue-type plasminogen activator. Stroke 2017;48:1376-85 CrossRef Medline

12. Campbell BC, Mitchell PJ, Kleinig TJ, et al; EXTEND-IA Investigators. Endovascular therapy for ischemic stroke with perfusion-imaging selection. N Engl J Med 2015;372:1009-18 CrossRef Medline

13. Berkhemer OA, Fransen PS, Beumer D, et al. A randomized trial of intraarterial treatment for acute ischemic stroke. N Engl J Med 2015; 372:11-20 CrossRef Medline

14. Seners $\mathrm{P}$, Hurford R, Tisserand $\mathrm{M}$, et al. Is unexplained early neurological deterioration after intravenous thrombolysis associated with thrombus extension? Stroke 2017;48:348-52 CrossRef Medline 